\title{
An Interleaver Design Algorithm based on a Cost Matrix for Turbo Codes
}

\author{
Didier Le Ruyet \\ Laboratoire Signaux et Systèmes \\ Conservatoire National des Arts et \\ Métiers \\ 75141 Paris Cedex 03, France \\ e-mail: leruyet@cnam.fr
}

\author{
Hong Sun \\ Departement Electronic and \\ Information Engineering \\ Huazhong University of Science \\ and Technology \\ 430074 Wuhan, China \\ e-mail: hsun@hust.edu.cn
}

\author{
Han Vu Thien \\ Laboratoire Signaux et Systèmes \\ Conservatoire National des Arts et \\ Métiers \\ 75141 Paris Cedex 03, France \\ e-mail: vu-thien@cnam.fr
}

Abstract - In this paper the design of interleavers for Turbo Codes is considered. The proposed algorithm is based on a Hamming weight cost matrix. It optimizes both the minimal distance of Turbo Codes and the passing of extrinsic information. Simulation results show that for short lengths these interleavers improve the error performances at high SNR.

\section{INTRODUCTION}

It is admitted that the interleaver is the key element of Turbo Codes [1] [2]. In order to optimize the distance spectrum and the minimal distance of Turbo Codes, the interleaver should map input sequences $u(D)$ which generate low weight output sequences $y_{1}(D)$ with interleaved sequences $v(D)$ which generate high weight output sequences $y_{2}(D)$, and vice versa. Due to the iterative structure of the turbo decoder, the interleaver should also guarantee a good passing of extrinsic information from one decoder to the other. The proposed interleaver optimizes both these two criteria. In order to increase the minimal distance, a Hamming weight cost matrix is used for the construction. The second goal is achieved since the proposed interleaver belongs to the family of cycle optimized interleavers [3]. The interleaver is built element by element using a tree search method.

Let $\boldsymbol{u}=\left[u_{0}, u_{1}, \ldots, u_{N-1}\right]$ and $\boldsymbol{v}=\left[v_{0}, v_{1}, \ldots, v_{N-1}\right]$ respectively be the input and output sequences of the interleaver. We have the relation : $\boldsymbol{v}=\boldsymbol{u} I$ where $I=\left\{a_{i j}\right\}_{N \times N}$ with $a_{i j} \in\{0,1\}$ and $\sum_{j} a_{i j}=\sum_{i} a_{i j}=1$. We can also define the interleaver with the permutation vector $E=$ $[e(0), e(1), e(2), \ldots, e(N \Leftrightarrow 1)]$ where $e(i)=j \Leftrightarrow a_{i j}=1$

\section{INTERLEAVER DESIGN}

For the construction of $E$, we will use a cost matrix $J$ of same dimension as $I . J=\left\{b_{i j}\right\}_{N \times N} b_{i j}$ is equal to the Hamming weight of the lowest Hamming weight code generated from the input sequences $u(D)$ with Hamming weight $w \leq w_{M A X}$ and supposing $a_{i j}=1$. Each new element $e(n)$ is chosen according to both criteria defined previously.

1. initialization :

$$
\begin{aligned}
& b_{i j}(0)=+\infty \quad \forall i, j \\
& e(0) \text { is chosen randomly }
\end{aligned}
$$

2. for $(n=1,2, \ldots, N \Leftrightarrow 1)$ : -update of $b_{i j}(n) \quad(i \geq n) \quad \forall j$ :

$$
b_{i j}(n)=\min \left[b_{i j}(n \Leftrightarrow 1), \min _{\mathcal{C}}\left(w+\sum_{k=0}^{N-1} y_{1 k}+\sum_{k=0}^{N-1} y_{2 k}\right)\right]
$$

$$
\begin{gathered}
\text { with } \mathcal{C}=\left\{u(D)=D^{l_{0}}+D^{l_{1}}+D^{l_{2}}+\cdots+D^{l_{w-1}}\right\} \\
w=\sum_{k=0}^{N-1} u_{k} \leq w_{M A X} \quad \text { and } \quad w \leq n+1 \\
\text { with } \quad l_{0}<l_{1}<\cdots<l_{w-3}<n \Leftrightarrow 1, \\
l_{w-2}=n \Leftrightarrow 1, \quad l_{w-1}>n \Leftrightarrow 1
\end{gathered}
$$

$e(n) \in \mathcal{E}=\mathcal{E} 1 \cap \mathcal{E} 2 . e(n)$ is chosen randomly in $\mathcal{E}$.

Equations (1) and (2) reduce the set $\mathcal{C}$ of input sequences $u(D)$ to test for each $n$. Equation (3) corresponds to the minimal distance constraint. Equation (4) corresponds to the cycle optimized constraint which imposes that two bits separated by $X$ bits $(X \leq L \Leftrightarrow 2)$ in the input sequence $u(D)$ should be separated by at least $L \Leftrightarrow 2 \Leftrightarrow X$ bits in the sequence $v(D)$. $\mathcal{E}$ is the set of all the new positions satisfying both constraints. This method allows us to build an interleaver with minimal distance $D_{M A X}$ and minimum cycle $L$. From [3], it is possible to build an interleaver with $L<\sqrt{N}+2$. If the tree search fails $(\mathcal{E}=\varnothing)$, the procedure should be started again. To obtain an interleaver with the greatest minimal distance, the procedure must be repeated by increasing the value $D_{M A X}$ until it is no longer possible to build the interleaver.

\section{Results And Conclusion}

Simulations using a $\mathrm{R}=1 / 3$ Turbo Codes with two 8 states RSC's with generator $(15 / 17)_{8}$ were performed. For $N=105$ bits, the parameters obtained with $w_{M A X}=3$ are $D_{M A X}=19$ and $\mathrm{L}=10$. Simulation results show that, at high SNR, the performances of Turbo Codes using this interleaver are $0.1 \mathrm{~dB}$ better than the best interleavers available in the literature [4].

\section{REFERENCES}

[1] C. Berrou, A. Glavieux, P. Thitimajshima., "Near Shannon limit error correcting coding and decoding : Turbo-codes," Proc. ICC'93, Geneva, Switzerland, pp. 1064-1070, May. 1993.

[2] S. Benedetto and G. Montorsi, "Unveiling Turbo Codes: Some Results on Parallel Concatenated Coding Schemes," IEEE Trans. Inform. Theory, vol. 42, No. 2, pp. 409-428, March 1996.

[3] D. Le Ruyet and H. Vu Thien, "Design of Cycle Optimized Interleavers for Turbo Codes," submitted to Int. Symp. on Turbo Codes and Related Topics, Brest, France, Sept. 2000.

[4] D. Divsalar and F. Pollara, "Weight distributions for Turbo Codes using random and nonrandom permutations," TDA Progress Report, 42-123, JPL, pp. 56-65, Aug. 1995. 